'Departamento Psiquiatría, Campus Oriente, Facultad de Medicina, Universidad de Chile ¿Unidad de Salud Mental, Instituto Nacional de Salud y Bienestar, Helsinki, Finlandia ${ }^{3}$ Dirección de Salud,

Municipalidad de Santiago.

Financiamiento: No se recibió financiamiento para este estudio.

Recibido el 27 de octubre de 2015, aceptado el 5 julio de 2016.

Correspondencia a: Dr. Pedro Retamal C. Departamento Psiquiatría, Campus Oriente. Av. Salvador 486, Santiago. pretamal@vtr.net

\section{Salud mental en Chile y Finlandia: desafíos y lecciones}

\author{
PEDRO RETAMAL C. ${ }^{1}$, NIINA MARKKULA ${ }^{1,2}$, SEBASTIÁN PEÑA ${ }^{2,3}$
}

\section{Mental health in Chile and Finland. Challenges and lessons}

This article analyses and compares the epidemiology of mental disorders and relevant public policies in Chile and Finland. In Chile, a specific mental health law is still lacking. While both countries highlight the role of primary care, Finland places more emphasis on participation and recovery of service users. Comprehensive mental health policies from Finland, such as a successful suicide prevention program, are presented. Both countries have similar prevalence of mental disorders, high alcohol consumption and high suicide rates. In Chile, the percentage of total disease burden due to psychiatric disorders is $13 \%$ and in Finland $14 \%$. However, the resources to address these issues are very different. Finland spends $4.5 \%$ of its health budget on mental health, while in Chile the percentage is $2.2 \%$. This results in differences in human resources and service provision. Finland has five times more psychiatric outpatient visits, four times more psychiatrists, triple antidepressant use and twice more clinical guidelines for different psychiatric conditions. In conclusion, both countries have similar challenges but differing realities. This may help to identify gaps and potential solutions for public health challenges in Chile. Finland's experience demonstrates the importance of political will and long-term vision in the construction of mental health policies.

(Rev Med Chile 2016; 144: 930-935)

Key words: Comparative Study; Delivery of Health Care; Mental Health; Public Policy.

\section{C} hile y Finlandia son países que comparten desafíos en salud mental: alta carga de enfermedad de salud mental, altas prevalencias de depresión, tasas de suicidio y consumo de alcohol.

Finlandia es un país nórdico de alto ingreso que en Chile se conoce particularmente por sus resultados en educación. El producto interno bruto (PIB) es casi el doble de Chile (38.569 PPP-ajustados USD per cápita vs 21.980 USD (PPP) en Chile, datos combinados de 2011-2015), pero la esperanza de vida en años es similar: 80,6 y 78,3 respectivamente, en tanto que el promedio OECD es $80,1^{1,2}$. Aunque ambos países pertenecen a la categoría de alto ingreso, Chile entró en la categoría 25 años después de Finlandia, y los países siguen teniendo diferentes niveles de ingreso.

Con el objetivo de comparar la situación de salud mental y, en especial, las políticas públicas de Chile y Finlandia, el presente artículo analiza la situación epidemiológica en salud mental y los mecanismos de políticas públicas sectoriales e intersectoriales diseñadas e implementadas en ambos países.

\section{Marco legislativo y político}

Finlandia cuenta con una Ley de Salud Mental (Mielenterveyslaki 116/1990) promulgada el año 1990. Esta ley se sustenta en las leyes para el "enfermo mental" (Mielisairaslaki) del año 1937, actualizada en el año 1977. En 1987 se decreta la ley 
para hospitales psiquiátricos de administración estatal, llegando finalmente a la ley integral de 1990. Desde entonces ha sido actualizada en múltiples ocasiones, siendo la última versión del año 2001.

El Plan Nacional de Salud Mental en Finlandia 2009-2015 se enfoca en: a) Reforzar la posición de los usuarios de servicios; b) Fortalecer la promoción de salud mental y c) Prevención y tratamiento de problemas de salud mental en todas las edades con un enfoque en atención primaria y comunitaria. Se busca, por ejemplo, proveer un servicio centralizado donde todos los servicios de salud y apoyo social están en un solo lugar; incluir a "expertos usuarios" en la planificación, implementación y evaluación de servicios; facilitar el reingreso al mercado laboral de personas con enfermedades psíquicas y la provisión de servicios en entornos diarios como establecimientos educacionales ${ }^{3}$.

En Chile el Plan de Salud Mental del año 2001 definió como elementos centrales: a) Promover la integración desde la promoción y prevención a lo curativo y rehabilitación; b) Establecimiento de redes de dispositivos territoriales, sumado a un rol activo de las organizaciones de usuarios y familiares y equipos de salud y c) Estimular la regulación de la calidad, financiamiento y recursos. También se ha buscado enfocar en la atención primaria de salud (APS). De las prioridades definidas en el plan, sólo cuatro condiciones de salud-depresión, esquizofrenia, abuso/dependencia de alcohol y drogas, y trastorno bipolar- fueron incluidos en el sistema de garantías explícitas de salud (GES) desde el 20054. Actualmente, el Ministerio de Salud está elaborando un nuevo plan de salud mental para los años 2016-2025, y una ley de salud mental, cuyos lineamientos principales aún no han sido dados a conocer.

Si bien ambos planes destacan el rol de la atención primaria como un nivel crucial en la provisión de servicios de salud mental, en el plan finlandés el énfasis está puesto en los usuarios, tanto en su participación como en la recuperación social y funcional.

\section{Epidemiología de salud mental comparada}

En Chile, según datos de cuatro regiones recolectados entre los años 1992 y 1999, la prevalencia de patología psiquiátrica en el último año es $22,6 \%$ (Tabla 1) ${ }^{5}$. En Finlandia, no hay un estudio comparable que incluya todas las categorías de enfermedades psiquiátricas, pero se ha estimado que $15-20 \%$ de los finlandeses han sufrido alguna enfermedad psiquiátrica en el curso de un año ${ }^{6,7}$. La prevalencia de trastornos depresivos (depresión y distimia) en los últimos 12 meses en Chile es $7,9 \%{ }^{8}$ y en Finlandia $9,6 \%{ }^{9}$. Sin embargo, la prevalencia de depresión en Chile en la última Encuesta Nacional de Salud parece ser más alta, $18,4 \%$, pero el instrumento utilizado para medirla es distinto que se ha usado en Finlandia y en la encuesta chilena anterior, lo que dificulta la comparación (Markkula et al., datos mandados a publicación).

Datos similares se pueden observar en el porcentaje de carga total de enfermedad del país que representan los trastornos psiquiátricos, que en Chile es $13 \%$ y en Finlandia $14 \%{ }^{10}$.

En ambos países, la tasa de suicidio es más alto

Tabla 1. Comparación de situación epidemiológica y recursos en salud mental en Chile y Finlandia

\begin{tabular}{|lcc|}
\hline & Chile & Finlandia \\
\hline Prevalencia de trastornos psiquiátricos en el último año & $22,6 \%$ & $15-20 \%$ \\
\hline Prevalencia de trastornos depresivos en el último año & $7,9 \%$ & $9,6 \%$ \\
\hline Porcentaje de carga total de enfermedad debido a trastornos psiquiátricos & $13 \%$ & $14 \%$ \\
Tasa de suicidio/100.000 habitantes & 13,3 & 16,1 \\
Gasto en salud (\% de PIB) & $7,4 \%$ & $9,4 \%$ \\
Gasto en salud mental (\% de presupuesto de salud) & $2,2 \%$ & $4,5 \%$ \\
Camas psiquiátricas por 1.000 habitantes & 2,2 & 5,3 \\
\hline Psiquiatras por 100.000 habitantes & 4,6 & 20 \\
\hline Uso de antidepresivos (dosis diarias por 1.000 personas) & 20 & 60 \\
Consultas en salud mental por 1.000 habitantes anual & 89 & 419 \\
\hline
\end{tabular}


que el promedio OECD $(12,4 / 100.000$ habitantes, tasa estandarizada): en Chile 13,3 y en Finlandia, 16,4 en $2013^{11}$. En promedio, la tasa de suicidio bajó entre 1985 y 2010 en la OECD, salvo en siete países, pero en Chile se observó el aumentó más alto después de Corea del Sur: de 7 en 1990 a 13,3 en 2013.

Cabe destacar que Finlandia tuvo una de las tasas de suicidio más altas en el mundo en fines de los años 1980, 50/100.000 habitantes en hombres. Frente a este problema, el Gobierno de Finlandia lanzó en 1987 un programa de prevención de suicidio, resultando en una disminución del número de suicidios, caída que ha seguido después del término del programa. El programa consistió en una etapa de investigación, en la cual se realizó una autopsia psicológica de todos los suicidios ocurridos durante el último año, y una etapa de implementación multisectorial. En esa etapa, se trabajó en seis subprogramas: desarrollar capacidades de actuar en situaciones de crisis en los colegios; trabajo de sensibilización de hombres en el servicio militar (obligatorio a todos los hombres finlandeses); desarrollar programas de prevención de suicidio a nivel regional y municipal; mejoramiento de calidad de cuidado de personas que intentaron suicidarse y desarrollo de una guía de tratamiento para atención primaria; colaboración con la iglesia luterana y desarrollo de una guía; y un estudio sobre el tema de suicidio en la prensa ${ }^{12}$. $\mathrm{Al}$ mismo tiempo que disminuyeron los suicidios, ha aumentado el consumo de los antidepresivos. Desde el año 1990, la tasa de suicidio en Finlandia ha bajado $47 \%$ (Figura 1).

En Chile, el peak en la tasa de suicidio se produjo en el año 2008 (13,4/100.000, taza estandarizada por sexo y edad), tras lo cual ha disminuido gradualmente. Si bien es posible plantear que dicha disminución es el resultado del mayor acceso a tratamiento de problemas de salud mental derivados del GES ${ }^{13}$, es importante mencionar que elnúmero total de consultas por depresión ha disminuido progresivamente en FONASA (Figura 2). Tanto en Chile como en Finlandia los factores socioeconómicos influyen en el suicidio ${ }^{14,15}$.

Finalmente, ambos países presentan riesgos futuros con posibles impactos en salud mental. Finlandia sigue en una crisis económica desde el 2008 con alto desempleo, y en Chile existe cierta inestabilidad económica y social con probables consecuencias negativas para la salud mental de la población, incluso podría impactar en aumento de los suicidios ${ }^{16}$. Sin embargo, el impacto de las crisis socioeconómicas en tasa de suicidio parece depender de las medidas del gobierno para aminorar el desempleo ${ }^{17}$.

\section{Recursos en salud mental y provisión de servicios}

Las similitudes en la epidemiología de los problemas de salud mental contrastan con las diferencias observadas en los sistemas de salud de ambos países. Esto incluye tanto la arquitectura general del sistema de salud como en la respuesta específica de los servicios de salud mental. En Finlandia, el aseguramiento y la provisión de servicios de salud son primordialmente públicos, financiado por impuestos generales, junto con el Fondo Nacional de Pensiones (KELA) que cubre algunos servicios de rehabilitación destinados a trabajadores (i.e. psicoterapia) y medicamentos.

La provisión pública de servicios implica que una enorme mayoría de los pacientes consulta en atención primaria, siendo derivados al nivel secundario según los protocolos establecidos en cada servicio de salud. Los servicios psiquiátricos son mayormente gratis para los usuarios, aunque a diferencia de Chile, la atención primaria de salud cuenta con enfermeras especializadas en psiquiatría y no psicólogos. Otra diferencia importante radica en que la entrega de medicamentos se

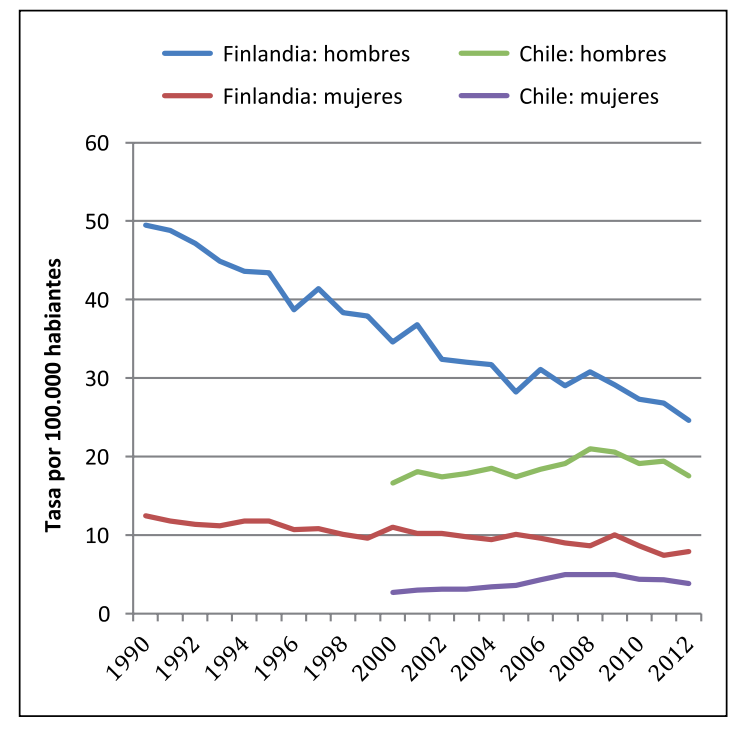

Figura 1. Tasa de suicidio en Chile y Finlandia 1990-2012. 


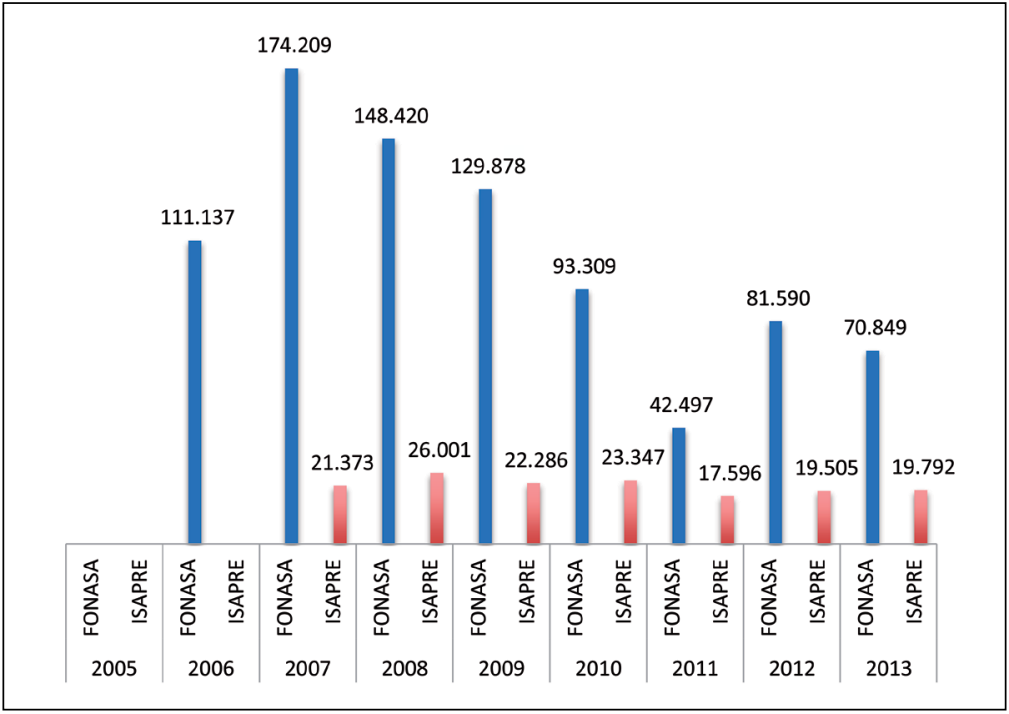

Figura 2. Consultas por depresión en FONASA y ISAPREs en Chile, 20062013. realiza en farmacias privadas donde, al momento del pago, se efectúa un descuento asociado a la cobertura de KELA. Esto implica que los médicos de atención primaria y secundaria tienen acceso a todo el arsenal terapéutico ambulatorio sin restricciones en las canastas de medicamentos. En forma anual, KELA entrega un informe a cada médico con estadísticas de sus propios patrones de prescripción comparados con sus pares.

En Chile un porcentaje de las personas con problemas de salud mental acceden a servicios gratuitos financiados por el estado, aquellas con patologías incluidas en el sistema de garantías explícitas de salud (GES), y en nivel A de FONASA. Sin embargo, todos los niveles de FONASA (A-D) acceden a atención gratis en atención primaria, donde existe atención en salud mental proveído por psicólogos.

Las mayores diferencias entre Chile y Finlandia se observan en los recursos que existen para la salud. El gasto en salud en Chile aumenta gradualmente: 6,4\% del PIB el año 2000 y el año 2014 es 7,4\% del PIB; aún lejos del gasto en Finlandia, que pasa de $6,7 \%$ a $8,7 \%$ en igual período ${ }^{2}$.

En Chile, el presupuesto de salud mental respecto del gasto total en salud habría aumentado hasta 3,1\% el año 2008, disminuyendo luego a $2,16 \%$ el $2012^{18}$. En comparación, en Finlandia los recursos para salud mental fueron $4,5 \%$ del presupuesto global de salud en $2010^{19}$.

Las mismas diferencias se pueden observar en cantidad de recursos humanos. Los países OECD tienen en promedio 15,6 psiquiatras por 100.000 habitantes. En Chile son sólo 5,8 en el sector público y 7,0 en el país, mientras que Finlandia tiene 20 por 100.000 habitantes ${ }^{18,19}$.

La falta de profesionales se traduce en diferencias en atenciones o consultas psiquiátricas. En Chile, en el año 2012 se realizaron 89 consultas de salud mental en atención primaria y secundaria por cada 1.000 habitantes, mientras en Finlandia fueron 419 consultas anuales por 1.000 habitantes $^{19,20}$.

En cuanto al uso de medicamentos, según OECD en Chile se emplean menos de 20 dosis diarias de antidepresivo por 1.000 personas por día. En Finlandia la cifra sube a más de 60, un poco más alto que el promedio de los países de la OECD, 56 dosis diarias ${ }^{19}$.

Para guiar los profesionales de salud y garantizar una atención de buena calidad para todos, en Finlandia se han creado varias guías clínicas. El proceso es coordinado por la Sociedad Médica Duodecim, y se invitan a los expertos más importantes del país en un trabajo voluntario. En psiquiatría existen guías nacionales para depresión, enfermedad bipolar, esquizofrenia, personalidad limítrofe, déficit atencional con hiperactividad, trastornos de alimentación, trastorno de estrés postraumático, tabaquismo, alcoholismo y abuso de sustancias, e insomnio ${ }^{21}$.

En Chile se dispone de guías clínicas para 
depresión, enfermedad bipolar, esquizofrenia, autismo, dependencia y abuso de alcohol y drogas en menores de 20 años. Estas guías son elaboradas por el Ministerio de Salud en base a recomendaciones de expertos.

\section{Problemas actuales}

En Chile la mayoría de las personas con problemas de salud mental no busca atención de salud, siendo esta brecha de cobertura más elevada en personas de menor estatus socioeconómico ${ }^{22}$. Es relevante considerar que en términos generales en la patología afectiva, dos tercios de las personas afectadas no buscan ayuda y de quienes consultan en APS la mitad no son diagnosticadas ${ }^{23}$.

Para mejorar la detección y tratamiento en atención primaria, sería importante capacitar a las personas trabajando en APS. Sin embargo, en Chile ha disminuido tanto el porcentaje de médicos capacitados en salud mental (de $23,1 \%$ a $18,6 \%$ ) como el porcentaje de centros con al menos un médico capacitado (de $65,1 \%$ a $57,4 \%$ ) entre 2004 y $2012^{18}$. Y si bien se incrementa el número de centros de atención no se ha traducido en un aumento de personas en tratamiento: las personas atendidas en centros de salud mental públicos baja de 1.633 personas por 100.000 beneficiarios el año 2004 a 1.573 por 100.000 beneficiarios el año 2012 y el tiempo de espera para la primera consulta psiquiátrica aumentó de 29 a 48 días. Sin embargo, el número total de recursos humanos dedicados a salud mental y psiquiatría en Chile en el sistema público ha aumentado en el mismo tiempo: el año 2004 existían 26,4 y el año 2012 aumentan a 41,5 profesionales y técnicos por 100.000 beneficiarios, con una tasa de psicólogos de17,1 por $100.000^{18}$.

En Finlandia, las personas con problemas de salud mental sufren distintos tipos de discriminación. La consecuencia más grave de eso es la mayor mortalidad de personas con trastornos psiquiátricos: personas con depresión tienen el doble riesgo de morir, que no se atribuye a comorbilidad somática ni comportamientos poco saludables como tabaquismo ${ }^{24}$. De hecho, las personas hospitalizadas en países nórdicos por enfermedades psiquiátricas, los hombres viven 20 años menos y las mujeres 15 años menos, comparadas con población general ${ }^{25}$. Como estos países disponen de buenos sistemas de salud, deberíamos suponer que en Chile las expectativas de vida para las personas con patología psíquica deberían ser aún más bajas, aunque no se cuenta con estudios que avalen esta hipótesis.

\section{Discusión y Conclusiones}

El presente artículo muestra que Chile y Finlandia, dos países con desafíos parecidos en salud mental, presentan recursos muy distintos para enfrentarlas necesidades de la población. Con prevalencias equivalentes de patologías psiquiátricas, alto consumo de alcohol y altas tasa de suicidio, en Finlandia se realizan casi cinco veces más atenciones o consultas psiquiátricas, hay cuatro veces más psiquiatras, triplica el uso de antidepresivos y duplica el número de guías clínicas para las diferentes condiciones psiquiátricas.

Las diferencias más relevantes en ambos países tienen relación con la cantidad de recursos destinados a salud mental, que se traduce en acceso a atenciones de especialista y a medicamentos. También se observan brechas en cuanto a la capacitación del personal de salud y en el desarrollo de guías clínicas, donde Finlandia ha desarrollado protocolos para un mayor número de enfermedades psiquiátricas. Chile, en cambio, posee acceso a profesionales con mayor grado de formación universitaria que Finlandia en el nivel primario de salud, donde las personas con problemas de salud mental son atendidas por enfermeras psiquiátricas.

Se observa en Finlandia un mayor desarrollo de políticas integrales de salud mental, destacando un programa exitoso de prevención del suicidio que podría ser extrapolado a Chile. Finlandia tiene una larga tradición de políticas para la reducción del consumo nocivo de alcohol destinadas a restringir la disponibilidad, asequibilidad y marketing de alcohol ${ }^{26}$; un marco legal así de coherente no existe en Chile, donde se ha avanzado de manera insuficiente.

Finalmente, se observan desafíos comunes no abordados relativos a la participación social de pacientes con patologías de salud mental y sus familias en la toma de decisiones y en el estigma asociado a la enfermedad psiquiátrica en donde ambos países requieren políticas públicas urgentes. Existen a su vez inequidades en cobertura de tratamiento en distintos grupos sociales que no han podido ser abordadas de manera efectiva.

En conclusión, el presente artículo demuestra 
que el uso de la comparación sistemática entre países con desafíos similares y realidades históricas, políticas y sociales distintas facilita identificar brechas y potenciales soluciones para los desafíos de salud pública en Chile. La experiencia de Finlandia demuestra la importancia de la voluntad política y la visión de largo plazo en la construcción de políticas de salud mental.

\section{Referencias}

1. World Bank. Data: GDP per capita, PPP 2015 [cited 2015 20.10.2015]. Available from: http://data.worldbank.org/indicator/NY.GDP.PCAP.PP.KD.

2. OECD. OECD Health Statistics 2015 [cited 2015 9.9.2015]. Available from: http://stats.oecd.org/.

3. Partanen AJ, Moring J, Nordling E, Bergman V. National Plan for Mental Health and Substance Abuse Work 2009-2015: From plan to action in 2009. 2010.

4. Minoletti A, Rojas G, Horvitz-Lennon M. Salud mental en atención primaria en Chile: aprendizajes para Latinoamérica. Cad Saúde Colet 2012; 20 (4): 440-7.

5. Vicente B, Kohn R, Rioseco P, Saldivia S, Baker C, Torres $\mathrm{S}$. Population prevalence of psychiatric disorders in Chile: 6-month and 1-month rates. Br J Psychiatry 2004; 184: 299-305.

6. Patana P. Mental Health Analysis Profiles (MhAPs): Finland. 2014.

7. Pirkola SP, Isometsa E, Suvisaari J, Aro H, Joukamaa M, Poikolainen K, et al. DSM-IV mood-, anxiety- and alcohol use disorders and their comorbidity in the Finnish general population-results from the Health 2000 Study. Soc Psychiatry Psychiatr Epidemiol 2005; 40 (1): 1-10.

8. Vicente B, Kohn R, Rioseco P, Saldivia S, Levav I, Torres S. Lifetime and 12-month prevalence of DSM-III-R disorders in the Chile psychiatric prevalence study. Am J Psychiatry 2006; 163 (8): 1362-70.

9. Markkula N, Suvisaari J, Saarni SI, Pirkola S, Peña S, Saarni S, et al. Prevalence and correlates of major depressive disorder and dysthymia in an eleven-year follow-up-results from the Finnish Health 2011 Survey. J Affect Disord 2015; 173: 73-80.

10. IHME. GBD Compare. In: Evaluation IfHMa, editor.: University of Washington; 2013.

11. OECD. OECD Health Statistics 2013 [cited 2015 9.9.2015]. Available from: http://www.oecd-ilibrary.org/ social-issues-migration-health/data/oecd-health-statistics_health-data-en.

12. Upanne M, Hakanen J, Rautava M. Can Suicide Be Prevented? The Suicide Project in Finland 1992-1996:
Goals, Implementation and Evaluation. Helsinki: 1999.

13. Salud Md. Guía Clínica Depresión en personas de 15 años y más. Santiago: 2013.

14. Mäki N, Martikainen P. Socioeconomic differences in suicide mortality by sex in Finland in 1971-2000 a register based study of trends, levels and life-expectancy differences. Scand J Public Health 2007; 35 (387).

15. Fullerton C, Retamal P, Salinas P, Mazuera P, Luengo J, Trebilcock JJ. Relación entre suicidio y el índice de desarrollo humano en Chile. Congreso Anual; Coquimbo: Sociedad Neurología Psiquiatría Neurocirugía 2010; 145-96.

16. Stuckler D, Basu S, Suhrcke M, Coutts A, McKee M. The public health effect of economic crises and alternative policy responses in Europe: an empirical analysis. Lancet 2009; 374 (9686): 315-23.

17. Chang SS, Stuckler D, Yip P, Gunnell D. Impact of 2008 global economic crisis on suicide: time trend study in 54 countries. BMJ (Clinical research ed) 2013; 347: f5239.

18. Minoletti A, Alvarado R, Rayo X, Minoletti M. Evaluación del sistema de salud mental en Chile. Informe sobre la base del Instrumento de evaluación del sistema de salud mental de OMS (OMS IESM / WHO AIMS). 2014.

19. Patana P. Mental Health Analysis Profiles (MhAPs): Finland. OECD Publishing: OECD, 2014.

20. WHO. World Mental Health Atlas 2014. Geneva: 2015.

21. Duodecim. Current Care Guidelines: Psychiatry Helsinki: Duodecim Medical Society; 2015 [cited 2015 7.8.2015]. Available from: http://www.kaypahoito.fi/ web/english/home.

22. Vicente B, Kohn R, Saldivia S, Rioseco P. Carga del enfermar psíquico, barreras y brechas en la atención de Salud Mental en Chile. Rev Med Chile 2007; 135: 1591-9.

23. Retamal P. Diagnóstico de Depresión Mayor por Médicos de Atención Primaria y Diferentes Especialidades Médicas. In: Meditarráneo E, editor. Enfermedades del Ánimo 2012.

24. Markkula N, Härkänen T, Perälä J, Partti K, Peña S, Koskinen S, et al. Mortality in people with depressive, anxiety and alcohol use disorders in Finland. Br J Psychiatry 2012; 200 (2): 143-9.

25. Wahlbeck K, Westman J, Nordentoft M, Gissler M, Laursen T. Outcomes of Nordic mental health systems: life expectancy of patients with mental disorders. $\mathrm{Br} \mathrm{J}$ Psychiatry 2011; 199: 453-8.

26. Mäkelä P, Österberg E. Weakening of one more alcohol control pillar: a review of the effects of the alcohol tax cuts in Finland in 2004. Addiction (Abingdon, England) 2009; 104 (4): 554-63. 